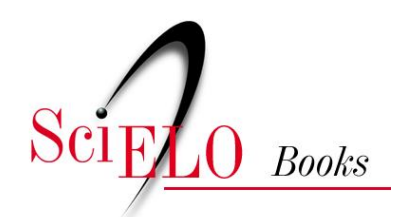

\title{
Experiencias de buenas prácticas \\ 14. La teoría del Neuroaprendizaje a través de las TIC en personas que asisten al servicio de rehabilitación
}

\author{
Juan Carlos Guachamín \\ Miriam Gallegos
}

\section{SciELO Books / SciELO Livros / SciELO Libros}

CARLOS GUACHAMÍN, J., and GALLEGOS, M. La teoría del Neuroaprendizaje a través de las TIC en personas que asisten al servicio de rehabilitación. In: GALLEGOS NAVAS, M., ed. La inclusión de las TIC en la educación de personas con discapacidad: relatos de experiencias [online]. Quito: Editorial Abya-Yala, 2019, pp. 181-190. ISBN: 978-9978-10-495-8. https://doi.org/10.7476/9789978104958.0017.

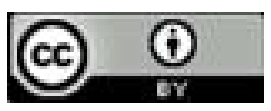

All the contents of this work, except where otherwise noted, is licensed under a Creative Commons Attribution 4.0 $\underline{\text { International license. }}$

Todo o conteúdo deste trabalho, exceto quando houver ressalva, é publicado sob a licença Creative Commons Atribição 4.0.

Todo el contenido de esta obra, excepto donde se indique lo contrario, está bajo licencia de la licencia Creative Commons Reconocimento 4.0. 
audiovisual, el periodismo y la fotografía. Esto se ha evidenciado a través del seguimiento de la coordinación, al equipo de producción de la revista.

Finalmente, se cuenta con cuatro revistas audiovisuales ${ }^{52}$ producidas por estudiantes sordos, con el apoyo de sus docentes, como material de sensibilización, educación y promoción de la lengua de señas y de los derechos de las personas sordas.

\section{La teoría del Neuroaprendizaje a través de las TIC en personas que asisten al servicio de rehabilitación}

"El primer movimiento de la pequeña mano hacia las cosas, el impulso de este movimiento, representa el esfuerzo del yo por penetrar en el mundo"

(María Montessori)

Centro de Rehabilitación

Ecuador

Quito

Responsable: Juan Carlos Guachamín

Gestores de la práctica: Esta experiencia se desarrolló con los miembros del equipo de rehabilitación (terapista físico, terapista del lenguaje).

Esta buena práctica gira en torno a actividades cotidianas desarrolladas a través de diferentes software los cuales aplicados de acuerdo a los intereses y necesidades de cada niño.

Se desarrollaron con una metodología altamente participativa, los profesionales utilizaron un gran abanico de posibilidades de estrategias didácticas para trabajar en varias áreas del conocimiento como pre matemática, funciones básicas, lenguaje, vocabulario, comprensión lectora.

La presente experiencia permite maximizar los resultados de la rehabilitación de los niños, niñas con trastornos neurológicos. Mediante el trabajo colaborativo con el equipo multimodal de la institución conformado por los profesionales en Terapia Física, Terapia de Lenguaje y Psicología Clínica. El entrenamiento neurocognitivo mediado por las TIC permite la modificación de las funciones cerebrales de manera temporal o permanente, así como de la conducta de los niños y niñas con trastornos neurológicos.

52 Estas revistas están disponibles a través del enlace al canal de YouTube de Fe y Alegría: https://goo.gl/qF2ynm 
182

\section{Motivación}

En todas las etapas de nuestra vida es posible modificar y cambiar la capacidad del cerebro, las experiencias de la vida le van moldeando, pero hay que tomar en cuenta que el momento de mayor plasticidad y donde se producen los mayores y más rápidos cambios es en la infancia.

El aprendizaje es la capacidad que tiene nuestro cerebro de adaptación a los requerimientos ambientales, el cerebro se desarrolla desde que nacemos hasta que morimos, Las experiencias que vivimos en nuestra niñez tienen un impacto decisivo en la "arquitectura" de nuestro cerebro. El cerebro de un niño/a de 3 años es dos veces más activo que el cerebro de un adulto con una elevada actividad intelectual. Por eso es fundamental ayudar a los niños/as en su desarrollo cerebral para que optimicen todas sus posibilidades a través del juego y del respeto a su identidad.

La maduración neurológica es diferente en cada niño/a, hay que respetar sus ritmos de maduración para ayudarles a desarrollarse y crecer con seguridad en sí mismos. En la edad adulta nuestro cerebro también cambia, tal y como lo usemos así se irá configurando.

A través del neuroaprendizaje se puede optimizar el funcionamiento del cerebro para potenciar los procesos de: atención, memoria, lenguaje, lectura, escritura, razonamiento y emociones.

\section{Objetivos}

Mejorar los niveles de comunicación y aprendizaje con la aplicación de las TIC y la participación activa de los padres de familia, de manera que se mitiguen las desventajas cognitivas a causa de las condiciones neurológicas de los niños que asisten al centro de rehabilitación.

\section{Desarrollo de la experiencia}

Consentimiento de todo el equipo de todo el equipo de trabajo (Terapia Física, Terapia de Lenguaje y Psicología Clínica,) con relación al uso de las TIC en la rehabilitación integral. 
Selección de la población beneficiaria a través de estudio de caso para conocer las potencialidades de cada persona.

Reunión con los padres de familia, asesorándoles sobre los beneficios de la terapia mediada por las TIC.

Ejecución del plan terapéutico individual, siguiendo los siguientes pasos:

Figura 94

Plan terapéutico individual
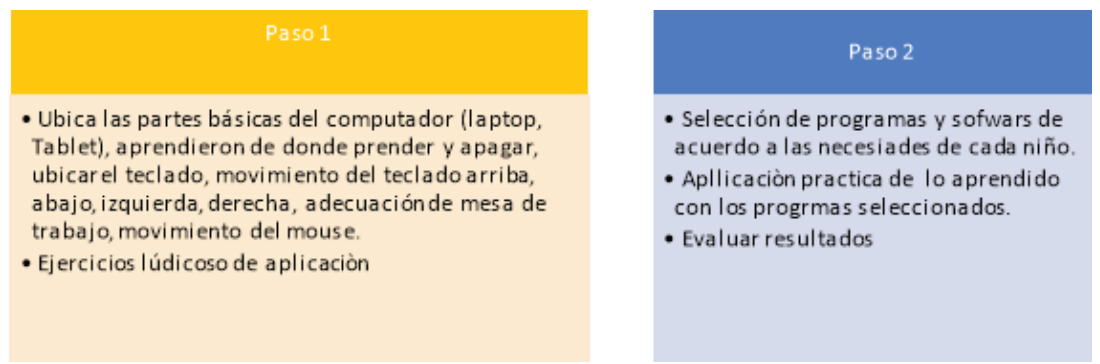

Fuente: Juan Carlos Guachamín (2017)

La fase terapéutica se desarrolla en dos momentos uno grupal y otro individual, los niños acuden a terapia tres días a la semana de los cuales se emplea 30 minutos de cada día en la aplicación de las TIC, donde se trabaja diferentes competencias con los software seleccionados que a continuación se detallan:

- Bouncy Balls

- Aplicaciones en la Tablet con Forge of neón

- Art of glow

- Kaleidoo, actividades causa-efecto 
184

Figura 95

Bouncy Balls

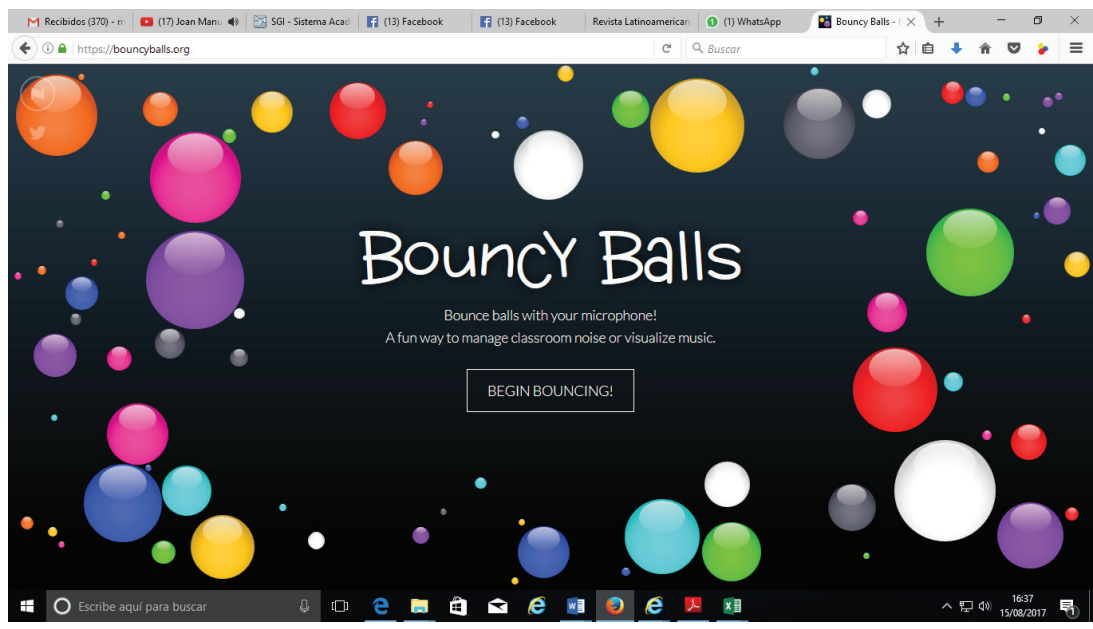

Fuente: Bouncy Balls

Recuperado de https://goo.gl/oT5T31

Posteriormente se aplica las TIC de manera individual respondiendo a las necesidades propias de condición neurológica, sus gustos, habilidades y nivel cognitivo.

Con Violeta y Sebastián, debido a su nivel cognitivo se prioriza el trabajo en el área de comunicación y matemáticas, con el siguiente software, los mismos que se pueden descargar de manera gratuita en los siguientes links: https://goo.gl/LvzycK; https:/goo.gl/uraqCr; https:// goo.gl/WdTzyk

Los software antes mencionados, permite trabajar las habilidades de comprensión lectora a través de la lectura con imagen y texto así como mediante emparejamiento de letras y nombres, actividades que se realizan en colaboración con la terapeuta de lenguaje. En cuanto a matemáticas (pre-matemáticas), el conocimiento de colores, formas, 
avanzamos hacia conocer números, contar, formar cantidades, y posteriormente a operaciones básicas suma y resta.

Figura 96

Ejemplos de uso del software
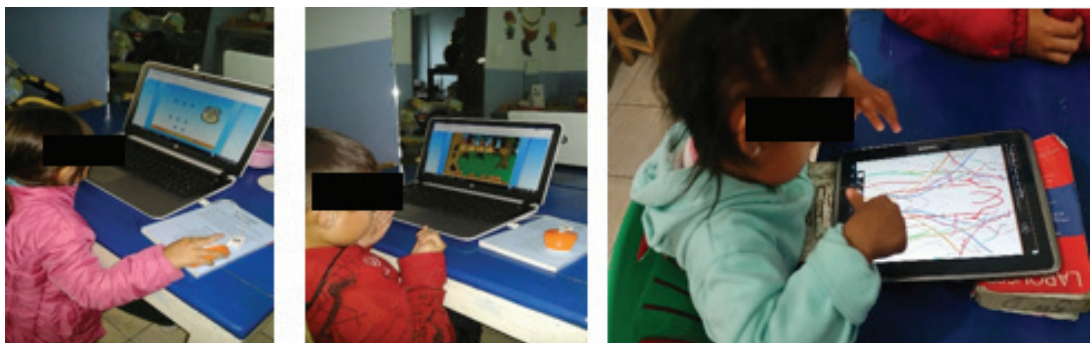

Fotos: Juan Carlos Guachamín (2017)

Por otro lado con Sofía, se adaptó un interruptor por presión, y uno de palanca para facilitar la manipulación del ratón, de igual manera se trabajó en Tablet para facilitar movimientos voluntarios de los dedos especialmente del índice y medio que posteriormente serán esenciales en la prensión de lápiz para la escritura.

Figura 97

Dispositivos adaptados
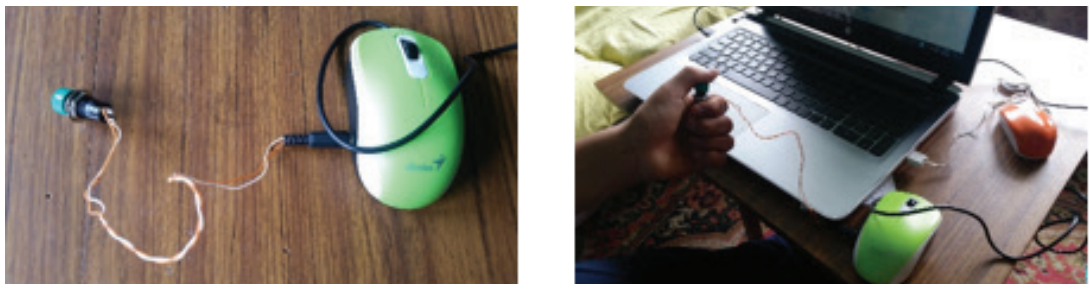

Fotos: Juan Carlos Guachamín (2017)

Para alcanzar el control cefálico de Sofía se utilizó el visor en 3D, se descarga la aplicación VR Ocean Aquarium 3D en el celular y posteriormente ubicarlo en el visor. VR Ocean Aquarium 3D es una apli- 
cación que trata sobre vida marina para lo cual se tiene que mover la cabeza, girando hacia arriba, abajo, a los lados y en forma circular poder ubicar a los peces que se encuentran a su alrededor, con este logramos movimientos voluntarios que fortalecerán por repetición los músculos del cuello. Una vez que mejoró en el control cefálico se la incorpora a trabajar pasturada adecuadamente en mesa facilitando actividades de aprendizaje y terapéuticas en mesa. Una vez pasturada en mesa, trabajamos autonomía y sensomotricidad, con el uso de audífonos, buscamos que discrimine sonidos, onomatopéyicos de animales domésticos, salvajes, medios de transporte, partes del cuerpo humano, apoyados con el profesional terapeuta de lenguaje. Las actividades que se realizó con Sofía en mesa son multisensoriales, y la finalidad es que al realizar una actividad motriz o táctil a la par participen otros sentidos como los visuales y auditivos, para esto se incorpora en el sentido auditivo la música bineural que solo es percibida por los oídos atreves de audífonos y su objetivo es que mediante notas musicales de alta vibración estimule las ondas cerebrales y mejore las conexiones nerviosas entre los hemisferios cerebrales, alcanzando estados de relajación, mejoramiento de la memoria, mayor grado de conciencia y vigilia, excelente grado de atención y concentración. La música bineural se la puede descargar en mp3 y tendrá su efecto solo con el uso de audífonos.

Figura 98

Sofía usando El visor 3D (segundo mes)
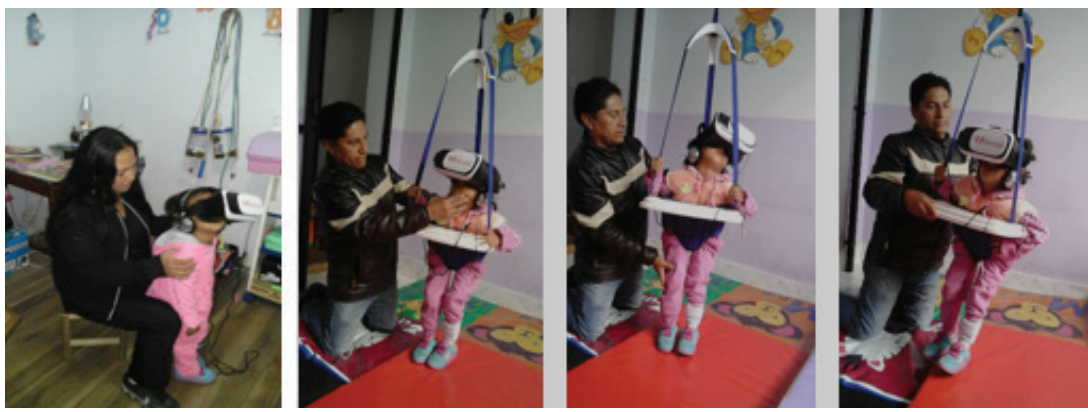

Fotos: Juan Carlos Guachamín (2017) 
VR. Ocean Aquarium 3D-Aplicando la música bineural
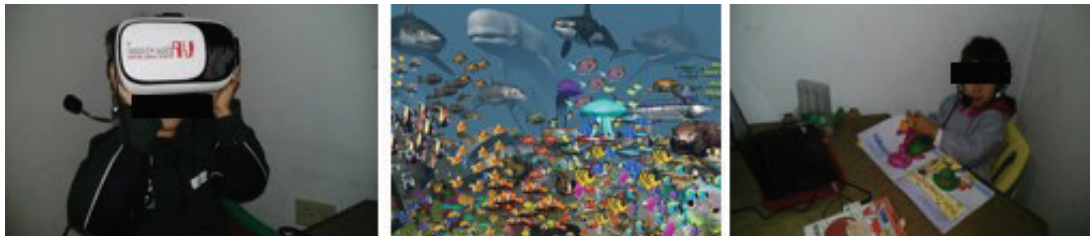

Fotos: Juan Carlos Guachamín (2017)

\section{Logros obtenidos}

Para la obtención de resultados fue necesario interpretarla mediante una metodología cuantitativa y cualitativa, pues de esa forma se detallan el progreso de cada niño (a) y sobre todo el desarrollo cognitivo a través de test y ejecución de programas adaptados. Cabe anotar que el test de inteligencia WIPPSI en este caso constituye una herramienta para la elaboración del plan de apoyo terapéutico.

\section{Interpretación cuantitativa}

Los resultados alcanzados en la aplicación del Test de Wechsler para menores de 6 años WPPSI a Violeta y Sebastián fueron:

\section{Tabla 9}

Resultados del diagnóstico inicial

\begin{tabular}{|c|c|c|c|}
\hline & Violeta 4 años 8 meses & $\begin{array}{l}\text { Sebastián } \\
\text { Edad de } 8 \text { años } 4 \text { meses }\end{array}$ & $\begin{array}{l}\text { Sofía } \\
\text { Edad } 3 \text { años } 2 \text { meses }\end{array}$ \\
\hline Verbal & $\begin{array}{l}22 \text { equivalente a } 65 \\
\text { Deficiente mental leve }\end{array}$ & $\begin{array}{l}19 \text { Equivalente a } 61 \\
\text { Deficiente mental leve }\end{array}$ & \multirow{3}{*}{$\begin{array}{l}\text { Test de Desarrollo de } \\
\text { Brunet -Lezine. Edad de } \\
\text { Desarrollo }\end{array}$} \\
\hline Ejecuciön & $\begin{array}{l}27 \text { equivalente a } 69 \\
\text { Deficiente mental Leve }\end{array}$ & $\begin{array}{l}39 \text { Equivalente a } \\
85 \text { Normal Torpe }\end{array}$ & \\
\hline Total & 49 equivalente a 63 & 58 Equivalente a 70 & \\
\hline C.I. & Deficiente mental leve & C.I. Limítrofe & $\begin{array}{l}\text { E.C }=38 \text { meses (Edad } \\
\text { Cronológica } \\
\text { E.D }=20 \text { meses (edad } \\
\text { de Desarrollo) }\end{array}$ \\
\hline
\end{tabular}

Fuente: Juan Carlos Guachamín (2017) 
Luego de 8 meses de recibir terapias, 3 veces a la semana, una hora diaria y de esta hora dedicarle 30 minutos en el Consultorio Terapéutico Infantil a la aplicación de las TIC; lo resultados tuvieron una mejoría considerable pues a continuación se detallas los resultados que obtuvieron los niños:

Tabla 10

Cuadro resumen de logros con relación al Coeficiente Intelectual

\begin{tabular}{|l|l|l|l|}
\hline Verbal & $\begin{array}{l}\text { Violeta } \\
4 \text { años } 8 \text { meses }\end{array}$ & $\begin{array}{l}\text { Sebastián } \\
\text { Edad d 8 años 4 meses } \\
\text { (Limítrofe) }\end{array}$ & $\begin{array}{l}\text { Sofía } \\
\text { Edad 3 años 2 meses }\end{array}$ \\
\hline Ejecución & $\begin{array}{l}36 \text { Equivalente a } \\
81 \text { (Normal Torpe) }\end{array}$ & $\begin{array}{l}\text { 29 Equivalente a } 74 \\
\text { (Limítrofe) }\end{array}$ & $\begin{array}{l}\text { Test de Desarrollo de } \\
\text { Brunet -Lezine. }\end{array}$ \\
\hline \multirow{2}{*}{ Total } & $\begin{array}{l}\text { 69 Equivalente a 91 } \\
\text { Coeficiente } \\
\text { Intelectual } \\
\text { Limítrofe }\end{array}$ & $\begin{array}{l}\text { E.C }=38 \text { meses } \\
\text { Coeficiente Intelectual } \\
\text { (Edad Cronológica) }\end{array}$ \\
\hline
\end{tabular}

Fuente: Juan Carlos Guachamín (2017)

Tabla 11

Cuadro resumen

\begin{tabular}{|l|l|l|l|}
\hline \multirow{3}{*}{ Antes } & $\begin{array}{l}\text { Violeta } \\
4 \text { años } 8 \text { meses }\end{array}$ & $\begin{array}{l}\text { Sebastián } \\
\text { Edad } 8 \text { años 4 meses }\end{array}$ & $\begin{array}{l}\text { Sofía } \\
\text { Edad 3 años 2 meses }\end{array}$ \\
\hline $\begin{array}{l}\text { Deficiente mental } \\
\text { leve }\end{array}$ & $\begin{array}{l}\text { 58 Equivalente a 70 } \\
\text { C.I. Limítrofe }\end{array}$ & $\begin{array}{l}\text { E.C=38 meses (Edad } \\
\text { Cronológica } \\
\text { E.D }=20 \text { meses (edad } \\
\text { de Desarrollo }\end{array}$ \\
\hline Después & $\begin{array}{l}\text { 69 Equivalente a 78 } \\
\text { Coeficiente }\end{array}$ & $\begin{array}{l}\text { 72 Equivalente a 80 } \\
\text { Coeficiente } \\
\text { Intelectual } \\
\text { Limítrofe }\end{array}$ & $\begin{array}{l}\text { E.C }=38 \text { meses (Edad } \\
\text { Cronológica } \\
\text { E.D }=26 \text { meses (Edad } \\
\text { de Desarrollo) }\end{array}$ \\
\hline
\end{tabular}

Fuente: Juan Carlos Guachamín (2017) 
189

\section{Interpretación cualitativa}

Los evaluados presentan un coeficiente mejor al inicial, lo que indica que al adaptarse al trabajo terapéutico a través de aplicación de las TIC. Demuestra un desarrollo relevante en sus capacidades ejecutivas en relación a las verbales, posee un CI global superior a la inicial, debido a destrezas en determinadas sub pruebas, demostrando mejor capacidad de comprensión, memoria a largo plazo, formación de conceptos, interés en el medio social y esfuerzo intelectual. Además de las funciones ejecutivas como mejor capacidad de percepción, visual, orientación espacial, usos de conceptos abstractos.

Indudablemente el trabajo profesional del equipo multimodal, Terapeuta de Lenguaje, Terapeuta Físico y Psicólogo Clínico, así como el apoyo de los padres de familia que creyeron en la propuesta de la aplicación de las TIC y ser testigos del progreso integral y rápido de sus hijos e hijas con trastornos neurológicos fue determinante para alcanzar los objetivos trazados.

La satisfacción de los padres de incluir a sus hijos e hijas a la escuela con las herramientas necesarias ha sido la mejor recompensa para el equipo profesional. Los resultados positivos alcanzados en esta experiencia profesional nos conducen a trabajar con otras personas con alteraciones neurológicas similares a la vez nos motiva compartir esta labor con otros profesionales o instituciones interesadas.

No se puede pasar por alto la ayuda profesional de los tutores que siempre estuvieron prestos para orientarnos, guiarnos especialmente sobre la aplicación de las tics adecuadas en cada caso. 
190

\title{
Recursos utilizados
}

\author{
Figura 100
}

Recursos utilizados en experiencia según el neuroaprendizaje

\section{RECURSOS HUMANOS}

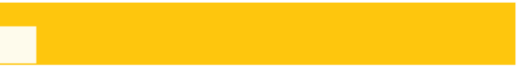

Equipos profesional

Niños niñas

familia
RECURSOS

MATERIALES

computador con pantalla táctil

tablet, VISOR 3D, Audífonos, sillas

correctivas, celular android

Fuente: Juan Carlos Guachamín (2017) 